\title{
Deposition Process of Sulfate and Elemental Carbon in Japanese and Thai Forests
}

\author{
Hiroyuki Sase*, Kazuhide Matsuda ${ }^{1)}$, Thiti Visaratana ${ }^{2)}$, Hathairatana Garivait ${ }^{3)}$, Naoyuki Yamashita, \\ Bopit Kietvuttinon $^{2)}$, Bundit Hongthong ${ }^{2)}$, Jesada Luangjame, Pojanie Khummongkol ${ }^{4)}$, Junko Shindo ${ }^{5}$, \\ Tomomi Endo ${ }^{6}$, Keiichi Sato, Shigeki Uchiyama, Masamitsu Miyazawa ${ }^{7)}$, Makoto Nakata ${ }^{7)}$ and \\ I. Wuled Lenggoro ${ }^{8)}$
}

\author{
Asia Center for Air Pollution Research (ACAP), Japan Environmental Sanitation Center, 1182 Sowa, Nishi-ku, Niigata 950-2144, Japan \\ ${ }^{1)}$ Tokyo University of Agriculture and Technology, 3-5-8 Saiwai-cho, Fuchu, Tokyo 183-8509, Japan \\ ${ }^{2)}$ Royal Forest Department, 61 Phaholyothin Road, Bangkok 10900, Thailand \\ ${ }^{3)}$ Environmental Research and Training Centre, Department of Environmental Quality Promotion Technopolis, Klong 5, \\ Klong Luang Pathumthani 12120, Thailand \\ ${ }^{4)}$ King Mongkut's University of Technology Thonburi, 240 Pracha U-thit Road Bangmod, Thungkru, Bangkok 10140, Thailand \\ ${ }^{5}$ National Institute for Agro-Environmental Sciences, 3-1-3 Kannondai, Tsukuba 305-8604, Japan \\ ${ }^{6}$ East Branch Office, Japan Environmental Sanitation Center, 10-6 Yotsuya-kamicho, Kawasaki-ku, Kawasaki, Kanagawa 210-0828, Japan \\ ${ }^{7)}$ Niigata University, 8050 Ikarashi 2-no-cho, Nishi-ku, Niigata 950-2181, Japan \\ ${ }^{8)}$ Tokyo University of Agriculture and Technology, Koganei, Tokyo 184-8588, Japan
}

*Corresponding author. Tel: +81-25-263-0559, E-mail: sase@acap.asia

\begin{abstract}
Particulate matter deposited on leaf surfaces may cause erosion/abrasion of epicuticular wax and the malfunction of stomata. However, the deposition processes of particulate matter, such as elemental carbon (EC), has not been studied sufficiently in Asian forest ecosystems. Deposition processes for particulate $\mathrm{SO}_{4}{ }^{2-}$ and EC were studied in a Japanese cedar forest in Kajikawa, Niigata Prefecture, Japan, and in a dry evergreen forest and a dry deciduous forest in Sakaerat, Nakhon Ratchasima province, Thailand. The $\mathrm{SO}_{4}{ }^{2-}$ fluxes attributed to rainfall outside the forest canopy (RF), throughfall (TF), and stemflow (SF) showed distinct seasonalities at both sites, increasing from November to February at the Kajikawa site and in March/April at the Sakaerat site. Seasonal west/ northwest winds in winter may transport sulfur compounds across the Sea of Japan to the Kajikawa site. At the Sakaerat site, pollutants suspended in the air or dry deposits from the dry season might have been washed away by the first precipitations of the wet season. The EC fluxes from RF and TF showed similar variations by season at the Kajikawa site, while the flux from TF was frequently lower than that from RF at the Sakaerat site. Particulate matter strongly adsorbed onto leaf surfaces is not washed away by rainfall and contributes to the EC flux. At the Kajikawa site, Japanese cedar leaf surfaces accumulated the highest levels of particulate matter and could not be neglected when calculating the total flux. When such leaf-surface particles were considered, the contribu-
\end{abstract}

tion of dry deposition to the total EC flux was estimated to be $67 \%, 77 \%$, and $82 \%$ at the Kajikawa site, and at the evergreen and deciduous forests of the Sakaerat site, respectively. Leaf-surface particles must be included when evaluating the dry and total fluxes of particulate matter, in particular for waterinsoluble constituents such as EC.

Key words: Throughfall, Particulate matter, Leaf, Cryptomeria japonica, Tropical species

\section{INTRODUCTION}

Forests in the East Asian region may suffer badly from the effects of air pollutants derived from the large energy consumption in the region. According to Ohara et al. (2007), emissions in Asia from 1986 to 2003 increased by $28 \%$ for black carbon, $119 \%$ for $\mathrm{SO}_{2}$, and $176 \%$ for $\mathrm{NO}_{\mathrm{X}}$. Although $\mathrm{SO}_{2}$ emissions in China peaked in 2006 and since then have gradually decreased, from 2000 to 2010, black carbon emissions have increased by $46 \%$ (Lu et al., 2011). Not only fuel combustion but also biomass burning is a large contributor to aerosol production, in particular in tropical regions (Badarinath et al., 2009). However, with the exception of a few studies (e.g., Matsuda et al., 2012, 2010), deposition processes of aerosols to Asian forests have not been studied extensively.

The forest canopy is an important interface between the atmosphere and forest ecosystems (Sase and Taka- 
matsu, 2009). Air pollutants including aerosols deposit on leaf surfaces or bark surfaces in the canopy. The leaf surface, in particular, is covered by a cuticle layer that plays an important role in preventing water loss, ion penetration, leaching, and fungal infection (Trunen and Huttunen, 1990). The deposition of air pollutants may cause physical and/or chemical alteration of the conditions on the leaf surface, thus resulting in physiological dysfunction of the trees (Sase et al., 1998). In fact, degradation of leaf-surface wax (epicuticular wax) by the deposition of particulate matter has been observed not only in European species, such as Picea abies (Grill and Golob, 1983) and Pinus sylvestris (Crossley and Fowler, 1986), but also in Asian species, such as Cryptomeria japonica (Sase et al., 1998). The changes to the leaf surface conditions accelerate cuticular transpiration (uncontrolled water loss) from the leaf surface (Sase et al., 1998) and dysfunction of stomata due to the water stress caused by particulate deposition (Takamatsu et al., 2001a). Moreover, degradation of wax and/or deposition of particulate matter caused an increase in the leaf-surface wettability, affecting ion fluxes in the canopy (Sase et al., 2008). Hence, it is important to study the deposition processes of air pollutants onto the forest canopy, in particular those of particulate matter.

Throughfall (TF) and stemflow (SF) are useful parameters for estimating the total deposition of ion constituents in forest areas, although the chemical compositions of TF and SF are affected strongly by canopy interactions, such as leaching, uptake, and/or consumption of ions on the tree surface (Shibata and Sakuma, 1996; Lovett et al., 1985). Collection of TF and SF may provide information on the dry deposition fluxes of certain ion constituents, such as $\mathrm{SO}_{4}{ }^{2-}$, whose canopy interactions are negligible (Lindberg and Lovett, 1992), although the possibility of canopy leaching of these ions has been suggested (Staelens et al., 2008). Particulate matter may account for almost half of the sulfur dry deposition in Niigata, Japan, (Endo et al., 2011) and Nakhon Ratchasima, Thailand (Fujimura et al., 2011), although the average ratio in the East Asian region was approximately $30 \%$ (Fujimura et al., 2011). Therefore, the flux of $\mathrm{SO}_{4}{ }^{2-}$ from $\mathrm{TF}+\mathrm{SF}$ may provide information about the dry deposition flux of the ion constituents of particulate matter in forest areas.

On the other hand, water-insoluble particulate matter, such as heavy metals and black carbon, which are strongly adsorbed onto leaf/bark surfaces, cannot be washed off completely by TF and/or SF. In fact, even after washing with de-ionized water in a laboratory, much of the heavy metals could still be extracted from the leaf surface of various Japanese species (Takamatsu et al., 2000). Therefore, in addition to TF and SF, par- ticulate matter strongly adsorbed onto leaf surfaces (leaf-surface particles) should be taken into account to explain the deposition process. Moreover, elemental carbon (EC), a major component of black carbon, may be chemically non-reactive in the forest canopy. This might be useful for clarifying the deposition processes of water-insoluble particulate matter.

The monsoon characterizes the climatic regime in the East Asian region and this play a role in the deposition patterns in the region. The area along the Sea of Japan suffers seasonal west/northwest winds in winter, and atmospheric deposition of TF and SF is increased (Kamisako et al., 2008). In a study of a tropical dry evergreen forest in northeast Thailand, where there is a clear dry season from November to February, it was reported that the vertical flux of nitrogen from the atmosphere through soil layers increased early in the wet season (Yamashita et al., 2010). Taking account of the climatic seasonality in the region, we selected two different types of forests in the East Asian region: a temperate coniferous forest along the Sea of Japan and a tropical dry forest in northeast Thailand.

In this paper, deposition processes of particulate $\mathrm{SO}_{4}{ }^{2-}$ and $\mathrm{EC}$ were investigated taking account of $\mathrm{TF}$, $\mathrm{SF}$, and/or leaf-surface particles in two types of forests in Japan and Thailand.

\section{METHODS}

\section{1 Study Sites}

The Kajikawa site is a Japanese cedar (Cryptomeria japonica) forest located in the northern part of Shibata city (formerly Kajikawa village), Niigata Prefecture, Japan, near the coast of the Sea of Japan (Kamisako et al., 2008). As of 2012, the forest was approximately 40 years old. A climate plot is shown in Fig. 1a), which was recorded at the Automated Meteorological Data Acquisition System (AMeDAS) in Nakajo (8 km north of the study site; Japan Meteorological Agency, 2012). The lowest monthly precipitation was $115 \mathrm{~mm}$ in April and there are perhumid conditions without a clear dry season throughout the year. The study site usually experiences snowfall from late December to March with the maximum depths exceeding $100 \mathrm{~cm}$, even on the slope.

The Sakaerat site is a dry evergreen forest (DEF) at the Sakaerat Environmental Research Station (SERS), Nakhon Ratchasima Province, northeast Thailand. A climate plot is shown in Fig. 1b), which was recorded at the Sakaerat Silvicultural Research Station in the same compound (Yamashita et al., 2010). The period between December and February is extremely dry, with monthly precipitation less than $50 \mathrm{~mm}$. In the period 

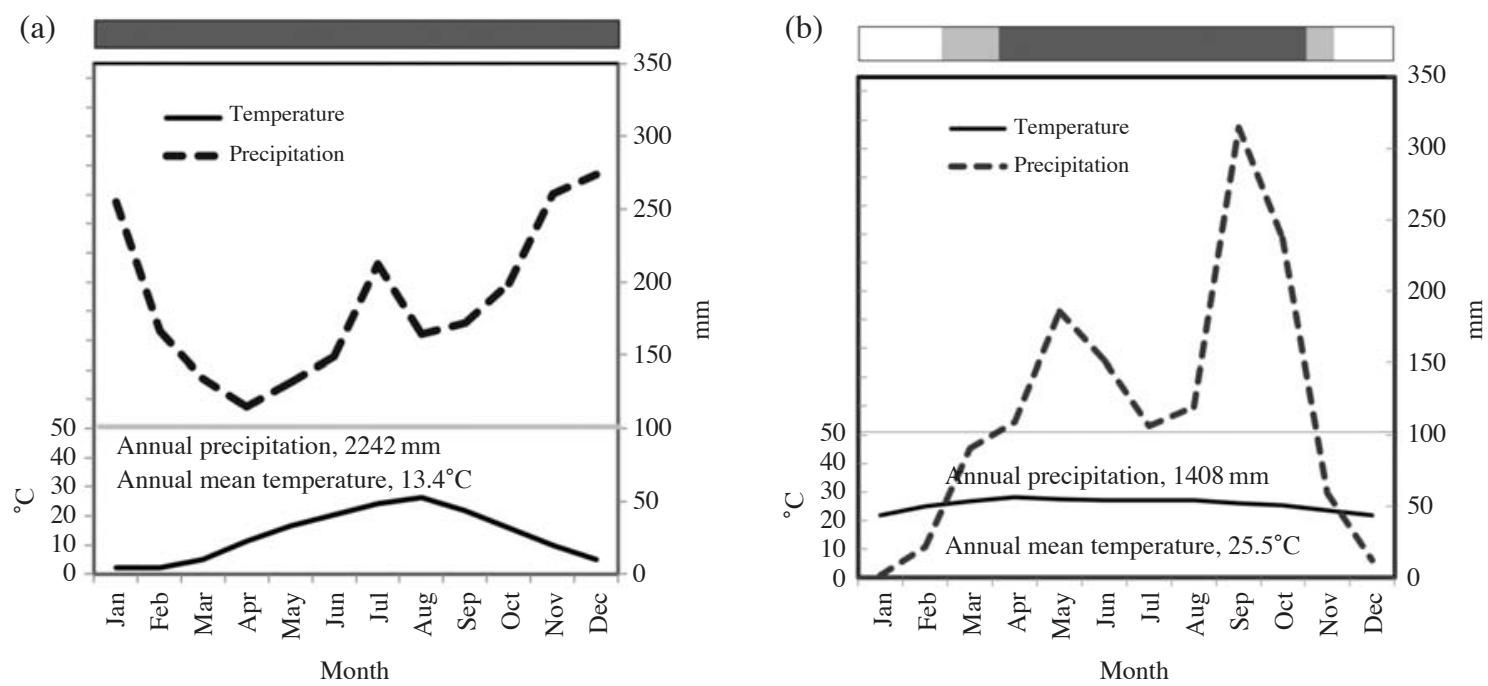

Fig. 1. Climate plots at (a) Nakajo AMeDAS near the Kajikawa site and (b) at the Sakaerat Silvicultural Research Station based on the data from 1981 to 2010 and from 2000 to 2008, respectively. Bars above the plots indicate the seasons: dark shade, perhumid period in wet season; light hatch, wet season; and white, dry season.

between April and October, there are perhumid conditions, with monthly precipitation exceeding $100 \mathrm{~mm}$. An observational tower for the measurement of dry deposition fluxes was constructed in a dry deciduous forest (DDF) in the same compound (Matsuda et al., 2012).

\section{2 Collection of Rainfall, TF and SF}

Six and five sets of samplers were installed for TF and SF measurements at the Kajikawa site and the DEF of the Sakaerat site, respectively. One and three samplers for rainfall outside the forest canopy (RF) were also placed in open spaces within the same areas of the Kajikawa and Sakaerat sites, respectively. TF and RF were collected using filtering-type bulk samplers with a funnel (200-mm diameter) at the Kajikawa site. A gutter type sampler $(0.1 \times 2 \mathrm{~m})$ and a bulk sampler with a funnel (200-mm diameter) were used at Sakaerat for $\mathrm{TF}$ and RF, respectively. The SF from trees was collected using flexible polyethylene foam $(30-\mathrm{mm}$ thickness) fitted around the trunk (Sase et al., 2008). The sample bottles were shaded from direct sunlight using aluminum foil to suppress algal growth while being kept in the field.

Rainwater samples (RF, TF, and SF) were collected at approximately 2 to 4 -week intervals from April 2002 and at 15-day intervals from October 2005 at the Kajikawa and Sakaerat sites, respectively. The volume of each sample was determined in the field and portions of each were taken to the laboratory for chemical analysis.

\section{3 Chemical analysis of precipitation samples}

The precipitation samples were stored in a refrigerator $\left(4^{\circ} \mathrm{C}\right)$ prior to chemical analysis in the laboratory. The $\mathrm{pH}$ was measured using a $\mathrm{pH}$ meter (HM-30V, TOA) and the electrical conductivity was measured using a conductivity meter (CM-40S, TOA). The samples were passed through a membrane filter (pore size, $0.45 \mu \mathrm{m}$ ) before ion analysis. The concentrations of inorganic ions were determined by ion chromatography (DX-100, -120, -500, ICS-1500 or 3000, DIONEX), while concentrations of $\mathrm{H}^{+}$ions were calculated from the $\mathrm{pH}$ values. The concentration was expressed as $\mathrm{mol}_{\mathrm{c}} \mathrm{L}^{-1}$. Ion fluxes (depositions) from $\mathrm{RF}$ and $\mathrm{TF}+\mathrm{SF}$ were determined by multiplying the concentration by the amounts of rainwater collected. The flux is expressed as $\mathrm{mol}_{\mathrm{c}} \mathrm{ha}^{-1}$ on a monthly or annual basis. In this paper, mainly the $\mathrm{SO}_{4}{ }^{2-}$ results will be discussed.

\section{4 Collection and Analysis of EC in RF and TF}

EC suspended in RF and TF was filtrated directly using a silica fiber filter (Advantec QR-100, $47 \mathrm{~mm}$ ) in the field. SF may also wash away EC, however the flux from SF may be significantly smaller than that from TF. In the case of the Kajikawa site, the contribution from SF was less than $8 \%$ of the total flux for most ion constituents (Sase et al., 2008). Therefore, SF was not collected for the EC flux in this study. At the Kajikawa site, existing filtering-type bulk samplers for $\mathrm{RF}$ and TF were used for collection of EC. One and three samplers were used for collection of EC from RF 
and TF, respectively, while representative samples in each period were analyzed for TF at the Kajikawa site. At the Sakaerat site, additional samplers equipped with filter holders were installed for RF and TF. A sampler for TF was also installed in the DDF of Sakaerat for comparison. Bi-weekly or monthly sampling was carried out continuously from January 2010 to January 2011. The filters collected from the forests were airdried and kept in a cool and dark place prior to analysis. The EC collected on the silica fiber filters was quantified using an OC/EC carbon analyzer (DRI Model 2001 or 2001A) with the Thermal Optical Transmittance (TOT) method. The precision by the OC/EC analyzer for atmospheric samples was on the order of 5-10\% (Chow et al., 1993). Collection efficiency of the filter including analytical errors in this study was estimated as approximately $80 \%$ (Matsuda et al., 2012). The EC flux is expressed in $\mathrm{mg} \mathrm{m}^{-2}$ on a monthly or annual basis.

\subsection{Extraction of Leaf-surface Particles}

The extraction of leaf-surface particles was conducted for several tree species at both sites; $C$. japonica in Kajikawa, the predominant three species (Shorea henryana, Hopea ferrea, and Dipterocarpus turbinatus) in the DEF, one of the predominant species (Pterocarpus macrocarpus) in the DDF, and a representative plantation species (Acacia mangium, an evergreen species) in Sakaerat.

The leaves were collected from branches that had been exposed to outer air. The $C$. japonica leaves were collected several times from branches at heights greater than $5 \mathrm{~m}$, and leaves were divided according to the foliating years, 2009, 2010, and 2011. The leaves, which seemed to be mostly one year or older, were collected from the DEF or A. mangium, although actual foliating time was unclear in the DEF in the tropical region. The P. macrocarpus leaves were collected at the end of the dry season just before defoliation. The leaves of the tropical species were collected at heights of 10-15 m by tree climbing in December 2009, February 2010, and March 2011. Leaves of A. mangium and P. macrocarpus were collected just once in February 2010 and those of D. turbinatus could not be collected in March 2011. The S. henryana leaves collected in March 2011 were divided into older and new leaves according to their positions on the branches.

Extraction of leaf-surface particles was conducted according to Takamatsu et al. (2001a). The leaf sample was washed with de-ionized water and gently dried on filter paper at room temperature. Leaf-surface particles were extracted using chloroform to dissolve epicuticular wax on the leaf surface for approximately $30 \mathrm{sec}$ and then collected on a silica fiber filter (Advantec $Q R$
-100). To make a homogeneous surface of particulate matter on the filter, a smaller filter $(25 \mathrm{~mm})$ was used for leaf-surface particles. The filters were air-dried and kept in a cool and dark place prior to analysis. $\mathrm{EC}$ on the filters was also determined using the OC/EC carbon analyzer. EC in the leaf-surface particles (leaf-surface EC) is expressed in $\mathrm{mg}$ per $\mathrm{m}^{2}$ of leaf surface area.

\section{6 Estimation of Annual Flux of EC in Forests}

Leaf-surface particles are deposited on the forest floor by defoliation. Therefore, the annual flux of EC to the forest floor is estimated as the sum of EC from TF and leaf-surface particles. In evergreen trees, leafsurface particles possibly remain in the forest canopy for several years until defoliation and finally dropping to the forest floor as a part of litterfall. In deciduous trees, leaf-surface particles are also deposited to the forest floor by litterfall. Therefore, the annual amount of litterfall should be taken into account to estimate the annual flux of leaf-surface particles to the forest floor. The mean amount of leaf litterfall in C. japonica forests in Japan is $350 \mathrm{~g} \mathrm{~m}^{-2}$ year $^{-1}$ (Kawahara, 1983), the amount of leaf litterfall measured in the DEF in Sakaerat was $530 \mathrm{~g} \mathrm{~m}^{-2}$ year $^{-1}$ (Yamashita et al., 2010), and that in the DDF was $536 \mathrm{~g} \mathrm{~m}^{-2}$ year-1 (Wachirinrat and Takada, 2003). These values were used to estimate the annual flux. The annual flux was calculated based on the amounts of EC on the leaf surface (per g dry leaf basis) and the leaf litterfall amounts.

\subsection{Quality Assurance}

Our laboratory participated in several inter-laboratory comparison projects to ensure and control the analytical quality of the data obtained (e.g., Network Center for EANET, 2010). Ion analysis, ion balances, and theoretical values of the electrical conductivity were assessed for all of the measured data according to the methods described in the Technical Manual of Acid Deposition Monitoring Network in East Asia (EANET) (EANET, 2000). In the case of Kajikawa site, RF samples generally had no significant error in the ion balances based on the EANET criteria, although occasional TF and SF samples had positive ion balance errors greater than the EANET criteria, which likely resulted from the presence of organic acid anions (Kamisako et al., 2008). In the case of Sakaerat site, many of RF samples had also positive ion balance errors greater than the EANET criteria, although analytical quality was checked regularly by reference samples. Both the ion balance and electric conductivity suggested the presence of unmeasured anions. In tropical savanna, organic acid anions derived from volatile organic carbons (VOCs) such as isoprene emitted by vegetation 

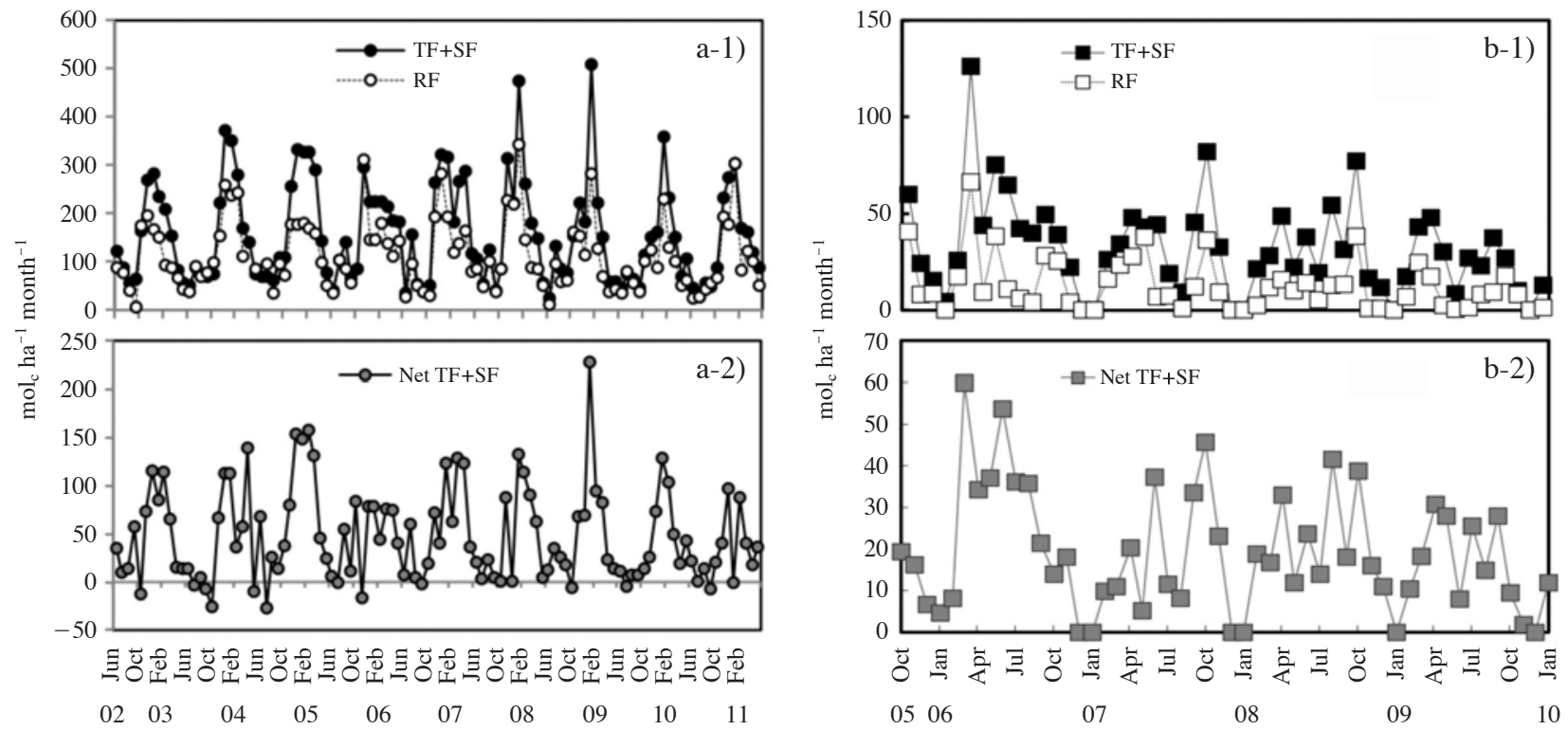

Fig. 2. (1) Seasonal variation of $\mathrm{SO}_{4}{ }^{2-}$ fluxes from rainfall outside the canopy and the sum of throughfall and stemflow and (2) net fluxes from throughfall and stemflow in (a) a Japanese cedar forest in Kajikawa, Japan, and (b) a dry evergreen forest in Sakaerat, Thailand. RF, rainfall outside the canopy; TF, throughfall; SF, stemflow. Net flux from TF+SF was calculated as the difference from RF.

(Sanhueza et al., 1989) and biomass burning (Sanhueza et al., 1992) may contribute to ion balance of rainwater. The similar positive ion balance errors can be seen in the EANET sites in tropical countries, including Nakhon Ratchasima site near Sakaerat site (EANET, 2011).

\subsection{Data Analysis}

Statistical analysis was conducted using $\mathrm{R}$ version 2.14.0 and the package "Rcmdr" version 1.7-3. The Wilcoxon signed-rank test and Spearman rank-order correlations were respectively used to compare the monthly fluxes from RF with those from $\mathrm{TF}+\mathrm{SF} / \mathrm{TF}$ and to assess any correlations. The Tukey's HSD test following one-way ANOVA was employed to compare the leaf-surface EC values of the tropical species.

Nine years of $\mathrm{SO}_{4}{ }^{2-}$ data for the Kajikawa site were summarized based on the water year defined as the time interval from June to May of the following year, and 4 years for the Sakaerat site based on the water year from February to January of the following year.

\section{RESULTS}

\section{3. $1 \mathrm{SO}_{4}{ }^{2-}$ Fluxes}

Seasonal variations in the $\mathrm{SO}_{4}{ }^{2-}$ fluxes are shown in Fig. 2 for 9 and 4 water years at the Kajikawa and Sakaerat sites, respectively. The monthly fluxes from $\mathrm{RF}$ and $\mathrm{TF}+\mathrm{SF}$ increased significantly in winter, from
Table 1. Mean annual $\mathrm{SO}_{4}{ }^{2-}$ flux at the Kajikawa and Sakaerat sites.

\begin{tabular}{|c|c|c|}
\hline & Kajikawa & Sakaerat \\
\hline & \multicolumn{2}{|c|}{$\mathrm{kmol}_{\mathrm{c}} \mathrm{ha}^{-1}$ year $^{-1}$} \\
\hline $\mathrm{RF}$ & 1.30 & 0.15 \\
\hline $\mathrm{TF}+\mathrm{SF}$ & 1.84 & 0.39 \\
\hline $\begin{array}{l}\text { Net } \mathrm{TF}+\mathrm{SF}^{* 1} \\
(\% \text { for } \mathrm{TF}+\mathrm{SF})\end{array}$ & $\begin{array}{l}0.54 \\
(29)\end{array}$ & $\begin{array}{l}0.24 \\
(62)\end{array}$ \\
\hline
\end{tabular}

Note. RF, rainfall outside canopy; TF+SF, sum of throughfall and stemflow. Mean annual fluxes were calculated based on the data for 9 and 4 water years at the Kajikawa and Sakaerat sites, respectively. The water years were defined as the time intervals from June to the following May and from February to the following January for the Kajikawa and Sakaerat sites, respectively. ${ }^{* 1}$, difference between RF and $\mathrm{TF}+\mathrm{SF}$.

November/December to February/March, at the Kajikawa site (Fig. 2a-1). At the Sakaerat site, the $\mathrm{SO}_{4}{ }^{2-}$ fluxes increased significantly in March/April at the beginning of the wet season and in October in the late wet season (Fig. 2b-1). The flux from TF+SF was generally larger than that from RF at both sites $(\mathrm{p}<0.001)$. The peak flux from $\mathrm{TF}+\mathrm{SF}$ was significantly larger at the Kajikawa site, almost 10 times that at the Sakerat site. The net flux from $\mathrm{TF}+\mathrm{SF}$, which was calculated as the difference from RF, showed a similar seasonality to those of RF and TF+SF (Fig. 2a-2 and b-2). The net flux from $\mathrm{TF}+\mathrm{SF}$ was generally positive, although 

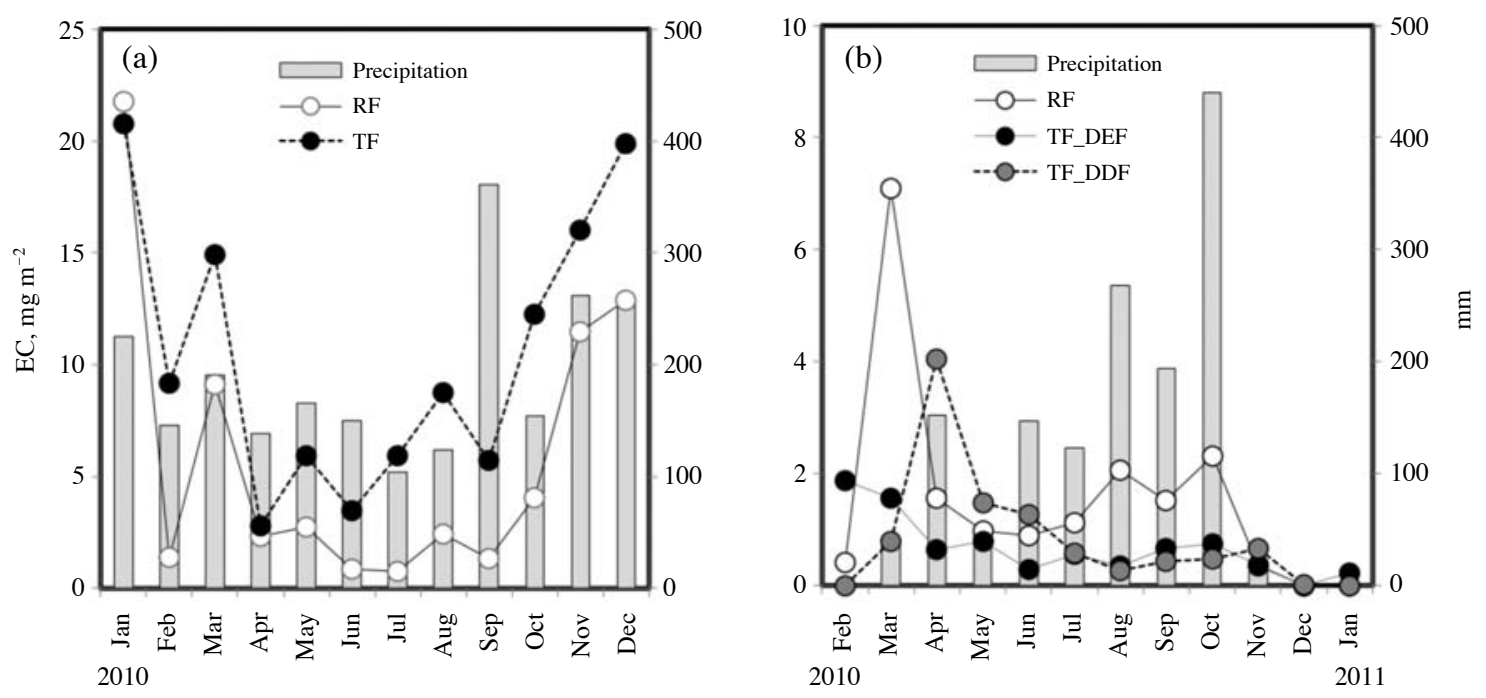

Fig. 3. Seasonal changes in elemental carbon deposition in (a) Kajikawa, Japan, and (b) Sakaerat, Thailand. EC, elemental carbon; DEF, dry evergreen forest; DDF, dry deciduous forest. RF and TF indicate EC depositions from rainfall outside the canopy and throughfall, respectively. Precipitation amounts were calculated on the basis of the rainwater from RF.
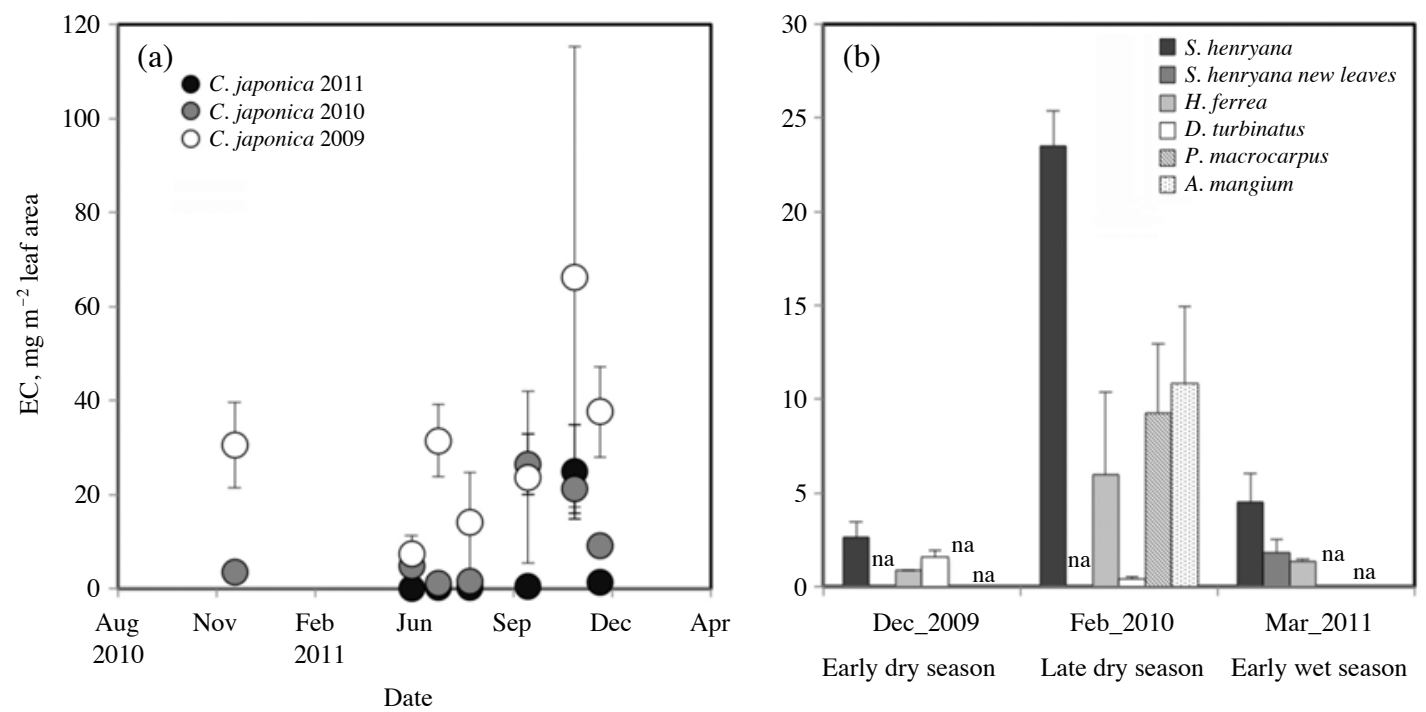

Fig. 4. Seasonal changes in the amounts of leaf-surface EC for different leaf ages in (a) Cryptomeria japonica and (b) tropical species. Years in (a) show the foliating year of the leaves. The "na" in (b) shows the species that were "not analyzed". The $S$. henryana leaves collected in March 2011 were divided into new and older leaves, while only older leaves were collected in other periods. Plots show the mean value and standard error of the triplicate analyses for each age in each season.

at the Kajikawa site it was negative at several points.

The mean annual fluxes of $\mathrm{SO}_{4}{ }^{2-}$ at the Kajikawa and Sakaerat sites are shown in Table 1. The annual flux from $\mathrm{TF}+\mathrm{SF}$ was larger than that from RF at both sites. The annual flux from $\mathrm{TF}+\mathrm{SF}$ was significantly larger at the Kajikawa site, 4.7 times larger than at the Sakaerat site.

\section{2 Fluxes of EC}

Fig. 3 shows seasonal variations of the EC fluxes with the precipitation for the same periods. At the Kajikawa site, the fluxes of EC from RF and TF increased in winter, in January and from October to December (Fig. 3a), which was similar to the variation of the $\mathrm{SO}_{4}{ }^{2-}$ flux. The flux from TF was generally higher than that from $\mathrm{RF}(\mathrm{p}=0.001)$. There was no relation- 
Table 2. Annual accumulation level of leaf-surface EC.

\begin{tabular}{|c|c|c|c|}
\hline Country & Forest type & Species & $\mathrm{EC}, \mathrm{mg} \mathrm{m}^{-2}$ leaf area, year ${ }^{-1}$ \\
\hline Japan (Temperate) & Plantation & C. japonica*1 & $26.8 \pm 5.5$ \\
\hline \multirow[t]{4}{*}{ Thailand (Dry tropical) } & $\mathrm{DEF}$ & $\begin{array}{l}\text { S. henryana } \text { h2 }^{2} \\
\text { H. ferrea }{ }^{* 2} \\
\text { D. turbinatus } * 2\end{array}$ & $\begin{array}{r}23.5 \pm 1.9^{\mathrm{a}} \\
6.0 \pm 4.4^{\mathrm{b}} \\
0.4 \pm 0.1^{\mathrm{b}}\end{array}$ \\
\hline & & Mean & $10 \pm 5.7$ \\
\hline & DDF & P. macrocarpus ${ }^{* 2}$ & $9.3 \pm 3.7^{\mathrm{ab}}$ \\
\hline & Plantation & A. mangium ${ }^{* 2}$ & $10.8 \pm 4.1^{\mathrm{ab}}$ \\
\hline
\end{tabular}

Note. The values were on leaf area basis. ${ }^{* 1}$ Mean with standard error (SE) $(n=33)$ for the leaves after autumn in 1-y and 2-y leaves; ${ }^{* 2}$ Mean with $\mathrm{SE}(\mathrm{n}=3)$ for the leaves in late dry season; Different letters beside values of Thai species indicate significant differences among the species by the Tukey's HSD test $(p<0.05)$ following one-way ANOVA.

ship between precipitation and the EC flux. At the Sakaerat site, the flux from RF significantly increased in March at the beginning of wet season, and slightly increased again from August to October, in the late wet season (Fig. 3b), which was also similar to the variation of the $\mathrm{SO}_{4}{ }^{2-}$ flux. The flux from TF in the DEF decreased from February to June and then was mostly stable, while the flux in the DDF showed a small peak in April and then decreased. The EC flux from RF was higher than the TF flux in several months; namely, in March at the beginning of the wet season and from July to October in the mid/late wet season. There was no significant difference between the fluxes from RF and TF in either the DEF or DDF. The flux from RF was positively correlated with the amount of precipitation $(\mathrm{R}=0.77, \mathrm{p}=0.02)$, while there was no correlation between the TF flux in the DEF/DDF and the amount of precipitation.

\section{3 EC in Leaf-surface Particles}

Seasonal changes in the amount of leaf-surface EC are shown in Fig. 4. Leaf-surface EC increased from spring to autumn and reached its maximum level in autumn in the current-year ( 0 -y, foliated in 2011) and previous-year (1-y, in 2010) leaves of $C$. japonica in Kajikawa, although the amounts dropped again in early winter especially in the 0 -y leaves (Fig. 4a). Following autumn, the amounts in the 1-y leaves became roughly constant within the range $20-40 \mathrm{mg} \mathrm{m}^{-2}$. In the tropical species, $S$. henryana and $H$. ferrea, which were collected three times, the largest leaf-surface EC was observed in the late dry season (Fig. 4b). For S. henryana, the leaf-surface EC was larger in older leaves than in new leaves in the early wet season. The leafsurface EC in P. macrocarpus, which was collected just once in the late dry season, was almost the same as that in A. manguim, S. henryana showed the highest level of leaf-surface EC of the species collected in each season.
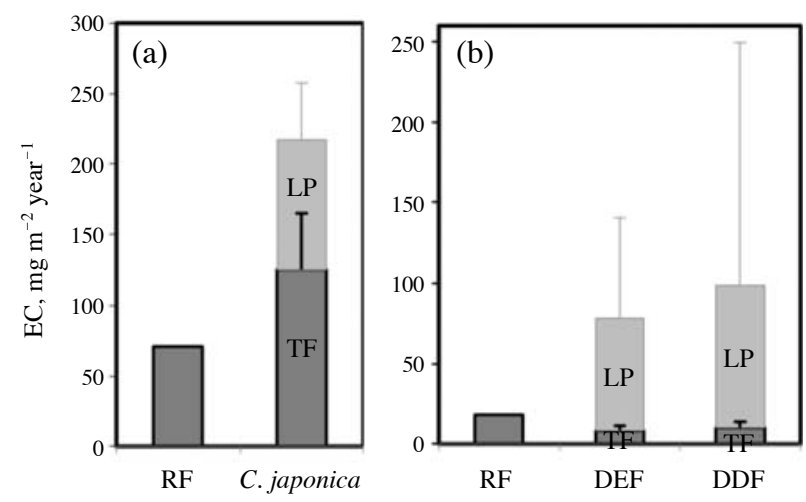

Fig. 5. Estimated annual fluxes of elemental carbon in (a) a C. japonica forest in Kajikawa, Japan, and (b) a dry evergreen /deciduous forests in Sakaerat, Thailand. RF, TF, and LP indicate elemental carbon (EC) fluxes from rainfall outside the canopy, throughfall, and leaf-surface particles, respectively. Error bars show $95 \%$ confidence intervals based on the uncertainties of spatial variation of $\mathrm{TF}$ and species/analytical variations of LP. Negative error bars are not shown here.

The annual accumulation levels of leaf-surface EC are summarized in Table 2. The amount of leaf-surface EC in C. japonica seemed to be high, although the values fluctuated as described above. The leaf-surface EC in $S$. henryana was significantly larger than that in $H$. ferrea $(\mathrm{p}=0.02)$ and D. turbinatus $(\mathrm{p}=0.004)$, while no significant difference was seen between other tropical species because of the data variation. The mean leafsurface EC of the three species in the DEF was $132 \pm$ $51 \mu \mathrm{g} \mathrm{g}^{-1}$ on a dry leaf basis. The values for $C$. japoni$c a$ and $P$. macrocarpus leaves were $262 \pm 56 \mu \mathrm{g} \mathrm{g}^{-1}$ and $166 \pm 66 \mu \mathrm{g} \mathrm{g}^{-1}$, respectively.

\subsection{Annual EC Flux in the Forests}

Annual fluxes of EC from RF, TF, and leaf-surface particles in the two forest areas are estimated in Fig. 5. At the Kajikawa site, the annual flux from TF was high- 
er than that from RF, reflecting the seasonality. When the flux from leaf-surface particles was added to that from TF to estimate a below-canopy total flux, the value was approximately $146 \mathrm{mg} \mathrm{m}^{-2}$ year $^{-1}$ larger than that from RF. At the Sakaerat site, the flux from TF was lower than that from RF in both the DEF and DDF. When the flux from leaf-surface particles was taken into account, the estimated total flux was larger than that from RF, approximately 60 and $81 \mathrm{mg} \mathrm{m}^{-2}$ year $^{-1}$ in the DEF and DDF, respectively. Uncertainties in the estimation are shown as error bars and will be discussed later.

\section{DISCUSSION}

\section{1 Fluxes from RF, TF, and SF}

As shown in Fig. 1a), the amount of precipitation at the Kajikawa site increased in winter from November to February and also in the rainy season from June to July. However, the seasonality of the $\mathrm{SO}_{4}{ }^{2-}$ and EC fluxes was clearer than this. The area along the Sea of Japan, where the Kajikwa site is located, is strongly affected by seasonal west/northwest winds in winter (Kamisako et al., 2008; Sase et al., 2008). Not only the precipitation pattern but also the seasonal winds in winter may determine the seasonal variation of the fluxes. In fact, $\mathrm{Na}^{+}$and $\mathrm{Cl}^{-}$, which are the major components of sea salt, exhibited quite similar seasonality (Kamisako et al., 2008). The seasonal winds through the Sea of Japan may transport air pollutants with sea salts from the Asian Continent. Ohizumi et al. (2001) estimated that almost half of the sulfur deposited in Niigata in winter was derived from coal combustion in China. It may be possible that EC derived from fossil fuel combustion was transported together with sulfur compounds.

For $\mathrm{SO}_{4}{ }^{2-}$, the net flux from TF+SF can be considered to come primarily from dry deposition (Lindberg and Lovett, 1992), although the possibility of leaching (Staelens et al., 2008) or uptake (Rautio and Huttunen, 2003) has also been suggested. The net flux from TF $+\mathrm{SF}$ in Kajikawa site $\left(27 \mathrm{mmol} \mathrm{m}^{-2}\right.$ year $^{-1}$, see Table 1) was comparable to the flux from dry deposition (approximately $25 \mathrm{mmol} \mathrm{m}^{-2}$ year $^{-1}$, Endo et al., 2011) calculated based on air concentrations and meteorological data at the Sado-seki EANET site, which is located approximately $90 \mathrm{~km}$ far from Kajikawa site, on an offshore island in the Sea of Japan. In the case of Sakaerat site, the flux was estimated as less than $10 \mathrm{mmol}$ $\mathrm{m}^{-2}$ year $^{-1}$ at the Nakhon Ratchasima EANET site (Fujimura et al., 2011) located approximately $5 \mathrm{~km}$ far from the study forest, which was slightly lower than the net flux from TF+SF but not so different $(12 \mathrm{mmol}$ $\mathrm{m}^{-2}$ year $^{-1}$, see Table 1). Therefore, the net fluxes from $\mathrm{TF}+\mathrm{SF}$ were discussed as contribution of dry deposition for $\mathrm{SO}_{4}{ }^{2-}$ in this paper. As for dry deposition of EC, studies in the East Asian region are very limited. According to Matsuda et al. (2012), the EC dry deposition in Sakaerat area, estimated by the inferential method based on measurements at the observational tower, was $0.58 \mathrm{mg} \mathrm{m}^{-2}$ day $^{-1}$ (approximately $211 \mathrm{mg} \mathrm{m}^{-2}$ year $^{-1}$ ). Since the value was significantly higher than the annual EC flux estimated from TF and leaf-surface particles (see Fig. 5b), appropriateness and uncertainties of the total and dry fluxes of EC were discussed below.

The $\mathrm{SO}_{4}{ }^{2-}$ flux from dry deposition at the Kajikawa site increased in winter (see Fig. 2a-2), similar to that from RF, which is considered mainly as wet deposition. It seems that seasonal winds through the Sea of Japan increased the concentration of air pollutants as well as the sea-salt component. In fact, the maximum monthly mean concentrations of $\mathrm{SO}_{2}$ and particulate $\mathrm{Na}^{+}$and $\mathrm{Cl}^{-}$were recorded in January and/or December at the Sado-seki EANET site (EANET, 2011). High wind speeds may also have contributed to an increase in deposition velocity (Endo et al., 2011; Erisman et al., 1997). Seasonal winds may also cause either an increase in air concentrations or deposition velocity resulting in high dry deposition in winter. Moreover, the forest canopy at the Kajikawa site is normally capped with snow in January and February. The surface may experience wetter conditions, although its roughness may decrease with snowcap. Wet conditions in the forest canopy may also enhance the flux from dry deposition, especially for $\mathrm{SO}_{2}$ (Matsuda et al., 2006).

At the Sakaerat site, the fluxes seem to be primarily regulated by the precipitation pattern. The EC flux from $\mathrm{RF}$ and the $\mathrm{SO}_{4}{ }^{2-}$ flux show similar seasonality to the amount of precipitation (see Fig. 2a). Air concentrations of many pollutants showed the opposite pattern, increasing at the end of the wet season and decreasing at the beginning of the wet season in the Nakhon Ratchasima EANET site in the same area (EANET, 2011). Biomass burning may peak during the dry period from January to March (Chang and Song, 2010). In fact, the higher values of particulate $\mathrm{SO}_{4}{ }^{2-}$ and EC were observed from February to April (EANET, 2010) and from January to March (Matsuda et al., 2012) in 2010, respectively. It was suggested that pollutants suspended in the air were flushed out by the first precipitations at the beginning of wet season. The first precipitations may also wash away ion constituents such as $\mathrm{SO}_{4}{ }^{2-}$ that accumulated on plant bodies during the dry season, and this results in a high flux from TF+ $\mathrm{SF}$ at the beginning of wet season. The net flux from $\mathrm{TF}+\mathrm{SF}$ showed the second peak in late wet season 
(Fig. 2b-2). Relative high deposition velocity until August (Matsuda et al., 2012) and/or low precipitation amounts in July/August may contribute to the flux from dry deposition. According to Matsuda et al. (2012), the EC dry deposition increased significantly from February to April in Sakaerat. However, the EC flux from TF only showed a small peak in April in the DDF (see Fig. 3b). This may be related to the adsorption of particulate matters onto the leaf surface, as discussed later.

\section{2 EC in Leaf-surface Particles}

Takamatsu et al. (2001b, 2000) reported that the amounts of heavy metals in leaf-surface particles increased with leaf aging from spring to winter and reached their maximum levels in winter. In our study, it seemed that leaf-surface EC stabilized after the autumn for 1-y leaves from the Japanese cedar (see Fig. 4). The lower values of leaf-surface EC in 1-y leaves before this may be related to changes in leaf-surface conditions due to significant meteorological events. Strong winds and rains may erode epicuticular wax on the leaf surface of $C$. japonica leaves and/or destroy its crystal structure (Sase et al., 1998). Strong seasonal winds in winter and also heavy rains in early summer may cause desorption of leaf-surface particles. Leaf-surface particles have been observed regularly in older leaves with degraded surface conditions (Sase et al., 2008, 1998). Deposition of particulate matter may also cause degradation of the wax structure (Grill and Golob, 1983). It is thought that particulate matter accumulates gradually on the leaf surface causing alteration of the surface condition.

In the tropical species, EC accumulated on the leaf surface during the dry season from December 2009 to February 2010 (see Fig. 4b). The significantly high dry deposition in February (Matsuda et al., 2012) may be the reason for the accumulation of EC on the leaf surface. Although actual causes of the lower values in March 2011 were not clear, it was demonstrated that EC accumulated more on the surface of older leaves of $S$. henryana than for new leaves. In the case of $P$. macrocarpus in the DDF, EC collected on the leaf surface just before defoliation in February 2010 may be the maximum level of deposition.

Japanese cedar accumulated the largest level of leafsurface EC among the species (see Table 2), even though the leaves were collected from branches at heights of around $5 \mathrm{~m}$. According to Takamatsu et al. (2000), more heavy metals such as Sb accumulate on leaves collected from upper branches than those from lower branches. This may be because the lower branches are sheltered from deposition (Mayer, 1993). Therefore, in the case of $C$. japonica, the value could be much larger if the leaves were collected from higher branches. The values would be informative not only for the estimation of dry deposition flux but also for the evaluation of the potential risk of the particulate matter to the plant species.

\section{3 Annual EC Flux in the Forests}

The contributions of dry deposition to the belowcanopy total EC flux, which were calculated as difference between RF and the sum of TF and leaf-surface particles, are $67 \%, 77 \%$, and $82 \%$ at the Kajikawa site, and the DEF and DDF at the Sakaerat site, respectively (see Fig. 5). According to Matsuda et al. (2012), the EC flux from dry deposition was $0.58 \mathrm{mg} \mathrm{m}^{-2}$ day $^{-1}$, which was determined using the air concentration and meteorological data at the observational tower in the $\mathrm{DDF}$ at the Sakaerat site. Compared to this value (approximately $211 \mathrm{mg} \mathrm{m}^{-2}$ year $^{-1}$ ), the flux due to dry deposition must have been underestimated here.

The collection efficiency of fine pure EC particles $(<100 \mathrm{~nm})$ by the silica fiber filter set in the filter holder was approximately $80 \%$ in this study (Matsuda et al., 2012). Moreover, overestimation of the flux may occur because of bulk sampling, which was assumed to be $10 \%$ (Matsuda et al., 2012). The errors above may occur either for RF or TF.

Spatial variation in the EC flux from TF may also be relatively large. Although spatial variation of the EC flux was not directly evaluated in this study, the mean spatial variation (relative standard variation, $\%$ ) of the $\mathrm{SO}_{4}{ }^{2-}$ flux from TF $(\mathrm{n}=5)$ was $32.8 \%$ for the last 4 years at the Sakaerat site, which was derived from gaseous $\left(\mathrm{SO}_{2}\right)$ or fine particulate (e.g., $\left.\left(\mathrm{NH}_{4}\right)_{2} \mathrm{SO}_{4}\right)$ matter. According to Huang and $\mathrm{Yu}$ (2008), the majority of the EC mass $(\cong 80 \%)$ existed in the fine mode. Therefore, we assumed that the contribution ratio of fine particulate matters to $\mathrm{SO}_{4}{ }^{2-}$ fluxes was constant in the study forest and the spatial variation of the EC flux was also approximately $33 \%$ at the Sakaerat site. The error bars for TF in Fig. 5 show confidence intervals based on the spatial variation. Even if the spatial variation is taken into account, the EC flux from TF may not exceed that from RF significantly. Therefore, not only TF but also the accumulation of EC on tree bodies, such as leaf and bark surfaces, should be taken into consideration when estimating the total/dry EC flux in the forest.

The amount of leaf-surface particles may vary significantly between species, as shown in Table 2. This may be another cause of the underestimation. Takamatsu et al. (2000) reported that the amounts of elements in leaf-surface particles were greater in conifers than in broad-leaf trees. Leaf-surface properties and/or canopy structures may affect the dry deposition pro- 
cess. Based on modeling of the particle deposition onto vegetation, Katata and Nagai (2010) suggested that the leaf area index (LAI) and/or leaf width had a significant effect on the dry deposition velocity and that a larger LAI and smaller leaf width may cause a larger deposition velocity. In fact, the leaves of $D$. turbinatus, which showed the lowest amounts of leaf-surface particles, were very large and almost twice as wide as those of other DEF species. Species variations, including analytical errors, are shown in Fig. 5 as the confidence intervals for the fluxes from leaf-surface particles. Since the sample number from the DDF was very limited, just once for $P$. macrocarpus, the estimated error is very large. It seems that the variation was one of the causes of the underestimation. Variation between species must be evaluated to estimate the EC flux more precisely in tropical mixed forests.

Litterfall amounts, which were used for the estimation of the EC flux, may vary depending on meteorological conditions. However, leaf biomass should be relatively stable in mature forests (Hachiya, 1983). The study forest in Kajikawa is 40 years old and considered to be sufficiently mature. Although the exact forest age is not clear in the natural forests in Sakaerat, the study forests are considered to have been relatively stable for at least the last several decades. Moreover, net leaf production of $C$. japonica was not significantly different between the low- and high-density forests (Hachiya, 1983). The estimate here using the litterfall may be informative as a trial calculation for the evaluation of the flux from leaf-surface particles, although fluctuations due to litterfall may occur.

In the case of deciduous trees, the amount of leafsurface particles in existing leaves just before defoliation may be equivalent to the annual flux, since all leaves fall to the forest floor annually. According to Matsuda et al. (2012), the LAI in the DDF was 2.5-3.5 on a single side of the leaves. When we applied the maximum LAI of 3.5 for our estimate, the contribution from dry deposition would be $57 \mathrm{mg} \mathrm{m}^{-2}$ year $^{-1}$, which was approximately $30 \%$ lower than the estimated value using litterfall $\left(81 \mathrm{mg} \mathrm{m}^{-2}\right.$ year $\left.^{-1}\right)$. However, this indicates that leaf-surface particles may contribute to the EC flux, even if the methods of estimation are different.

Bark on tree stems may also keep particulate matter on their surface (Hokura et al., 2009). The stem area index (SAI, total surface of stem to ground area) in tropical forests was estimated to be approximately 2.0; e.g., 1.7 in the Amazon (Chambers et al., 2004), 1.52.0 in the Amazon and/or Andes (Robertson et al., 2010). For the surface area of trees, the leaf area may be more than 3 times larger than the stem area, since the total LAI in the DDF was approx. 5.0-7.0 for both sides of leaves. The contribution of particulate matter on the bark surface may account for as much as $30 \%$ of leaf-surface particles, although we did not measure the accumulation rate of particulate matter on the bark surface and the EC flux from SF in this study.

Although the uncertainties discussed above, such as litterfall fluctuations and bark-surface particles, cannot be estimated precisely, it can be seen that leaf-surface particles made a contribution to the dry and total EC fluxes at the Sakaerat site.

The contribution ratio of leaf-surface particles to the EC flux by dry deposition may be smaller at the Kajikawa site than at the Sakaerat site, since the flux from TF was generally larger than that from RF. Since the amount of precipitation at the Kajikawa site is significantly larger than that of the Sakaerat site, leaf-surface particles may be washed off effectively by TF. Since the forest was covered mainly by one species, $C$. japonica, the spatial variation of the fluxes from $\operatorname{TF}(n=6)$ at the Kajikawa site was also smaller than those in Sakaerat; $18.8 \%$ for $\mathrm{SO}_{4}{ }^{2-}$ for the last 9 years. Even if the spatial variation is taken into account, the flux from TF is larger than that from RF (see Fig. 5). As shown in Table 2, EC can accumulate more on the leaves of $C$. japonica than on those of tropical species. Moreover, Takamatsu et al. (2000) reported that, of the five major Japanese coniferous species, the amounts of elements in leaf-surface particles were largest in C. japonica. Therefore, it is suggested that the contribution from leaf-surface particles to the total EC flux was not negligible at the Kajikawa site.

\section{CONCLUSIONS}

The deposition processes of particulate $\mathrm{SO}_{4}{ }^{2-}$ and $\mathrm{EC}$ were studied in different types of forests in the East Asian region. In the case of $\mathrm{SO}_{4}{ }^{2-}$, the monthly fluxes from RF and $\mathrm{TF}+\mathrm{SF}$ showed clear seasonality in both forests. The flux was mainly regulated by seasonal west/northwest winds in winter in the $C$. japonica forest at the Kajikawa site, while it was regulated by the precipitation pattern in the DEF at the Sakaerat site. It seems that dry deposition was an important process in both forests, in particular for sulfur, although the detailed contributions from particulate matter could not be evaluated. The method employed here using fluxes from TF and SF cannot explain the deposition processes of some important constituents, such as $\mathrm{NH}_{4}{ }^{+}$, because of their canopy interactions (Lovett, 1992), although $\mathrm{NH}_{4}{ }^{+}$can be considered to be one of the major counterparts of $\mathrm{SO}_{4}{ }^{2-}$ in the formation of particulate matter. However, the total $\mathrm{SO}_{4}{ }^{2-}$ flux from $\mathrm{TF}+\mathrm{SF}$ certainly reflects the dry deposition of particulate $\mathrm{SO}_{4}{ }^{2-}$, 
since the water-soluble constituent deposited on the plant body can be easily washed off by rainwater. In this study, a large contribution from dry deposition to the total $\mathrm{SO}_{4}{ }^{2-}$ flux was confirmed in the two forests.

In the case of EC, the monthly fluxes from RF and TF showed similar seasonality to those of $\mathrm{SO}_{4}{ }^{2-}$ at the Kajikawa site, while the seasonality in TF was not clear at the Sakaerat site. The EC flux from TF cannot adequately explain the deposition process in the forest area. The particulate matter deposited on the forest canopy may accumulate on plant bodies including the leaf surface (Takamatsu et al., 2000). Some of them are strongly adsorbed onto the leaf surface and cannot be washed off by precipitation. Such leaf-surface particles should be taken into account when evaluating the flux of particulate matter by dry deposition. Accumulation of leaf-surface particles may be harmful to plant physiology (Takamatsu et al., 2001a, b; Sase et al., 1998) and emission of particulate matter is still increasing in the East Asian region (Lu et al., 2011). The deposition process of particulate matter to the forest area must be further investigated to take account of the contribution from leaf-surface particles to the total flux and its effects on plants. Leaf-surface particles may have a larger contribution to the flux in the case of water-insoluble constituents, such as EC, than in the case of water-soluble ones, such as $\mathrm{SO}_{4}{ }^{2-}$.

\section{ACKNOWLEDGEMENT}

We thank the local government of Niigata Prefecture and Mr. Kohei Funayama and Mr. Takeo Funayama (managers of the area) for their permission to use the selected forest area in Kajikawa as a study site, and Prof. Shiro Hatakeyama and Prof. Takeshi Izuta, Tokyo University of Agriculture and Technology (TUAT) for their support on the project. The study was supported financially by the Grant-in-Aid for Scientific Research on Innovative Areas (20120012) from the Ministry of Education, Culture, Sports, Science, and Technology (MEXT), Japan, and by the Environment Research and Technology Development Fund (B-0801) from the Ministry of the Environment of Japan.

\section{REFERENCES}

Badarinath, K.V.S., Latha, K.M., Chand, T.R.K., Gupta, P.K. (2009) Impact of biomass burning on aerosol properties over tropical wet evergreen forests of Arunachal Pradesh, India. Atmospheric Research 91, 87-93.

Chang, D., Song, Y. (2010) Estimates of biomass burning emissions in tropical Asia based on satellite-derived data. Atmospheric Chemistry and Physics 10, 23352351.

Chambers, J.Q., Tribuzy, E.S., Toledo, L.C., Crispim, F., Higchi, N., dos Santos, J., Araújo, A.C., Kruijt, B., Nobre, A.D., Trumbore, S.E. (2004) Respiration from a tropical forest ecosystem: partitioning of sources and low carbon use efficiency. Ecological Applications 14(4) Supplement, S72-S88.

Chow, J.C., Watson, J.G., Pritchett, L.C., Pierson, W.R., Frazier, C.A., Purcell, R.G. (1993) The DRI thermal/ optical reflectance carbon analysis system: description, evaluation and applications in U.S. air quality studies. Atmospheric Environment 27, 1185-1201.

Crossley, A., Fowler, D. (1986) The weathering of Scots pine epicuticular wax in polluted and clean air. New Phytol. 103, 207-218.

EANET (2000) Technical Manual for Wet Deposition Monitoring in East Asia. Network Center for EANET, Acid Deposition and Oxidant Research Center, Niigata, Japan.

EANET (2011) Data Report 2010. Network Center for EANET, Asia Center for Air Pollution Research, Niigata, Japan.

Endo, T., Yagoh, H., Sato, K., Matsuda, K., Hayashi, K., Noguchi, I., Sawada, K. (2011) Regional characteristics of dry deposition of sulfur and nitrogen compounds at EANET sites in Japan from 2003 to 2008. Atmospheric Environment 45, 1259-1267.

Erisman, J.W., Draaijers, G., Duyzer, J., Hofschreuder, P., van Leeuwen, N., Römer, F., Ruijgrok, W., Wyers, P., Gallagher, M. (1997) Particle deposition to forestsSummary of results and application. Atmospheric Environment 31, 321-332.

Fujimura, Y., Matsuda, K., Sato, K., Ohizumi, T. (2011) Dry deposition estimation of sulfur oxides on forests in East Asia-Estimation of simplified methodology on deposition velocity estimation-. Earozoru Kenkyu 26(4), 286-295. (in Japanese with English summary)

Grill, D., Golob, P. (1983) SEM-investigations of different dust depositions on the surface of coniferous needles, and the effect on the needle-wax. Aquilo, Serie Botanica 19, 255-261.

Hachiya, K. (1983) Material production. In Sugi-no-subete (Everything about Japanese cedar, Sakaguchi, K. eds) National Forestry Extension Association of Japan (in Japanese), pp. 78-87.

Hokura, A., Chiba, H., Satake, K., Nakai, I. (2009) Development of a new sampling method using synthetic resin adhesive for environmental monitoring of heavy-metal deposits on tree bark. Bunseki Kagaku 58, 265-271. (in Japanese with English summary)

Huang, X.F., Yu, J.Z. (2008) Size distributions of elemental carbon in the atmosphere of a coastal urban area in South China: characteristics, evolution processes, and implications for the mixing state. Atmospheric Chemistry and Physics 8, 5843-5853.

Japan Meteorological Agency (2012) http://www.jma.go. jp/jma/indexe.html. 
Kamisako, M., Sase, H., Matsui, T., Suzuki, H., Takahashi, A., Oida, T., Nakata, M., Totsuka, T., Ueda, H. (2008) Seasonal and annual fluxes of inorganic constituents in a small catchment of a Japanese cedar forest near the Sea of Japan. Water, Air and Soil Pollution 195, 51-61.

Katata, G., Nagai, H. (2010) Recent trend and problems in modeling particle deposition onto vegetation. Earozoru Kenkyu 25(4), 323-330. (in Japanese with English summary)

Kawahara, T. (1983) Material cycles. In Sugi-no-subete (Everything about Japanese cedar, Sakaguchi, K. Eds) National Forestry Extension Association of Japan (in Japanese), pp. 87-99.

Lindberg, S.E., Lovett, G.M. (1992) Deposition and forest canopy interactions of airborne sulfur: results from the integrated forest study. Atmospheric Environment 26A, 1477-1492.

Lovett, G.M. (1992) Atmospheric deposition and canopy interactions of nitrogen, InAtmospheric deposition and forest nutrient cycling (Johnson, D.W., Lindberg, S.E. Eds), Springer-Verlag, New York. pp. 152-166.

Lovett, G.M., Lindberg, S.E., Richter, D.D., Johnson, D.W. (1985) The effects of acidic deposition on cation leaching from three deciduous forest canopies. Canadian Journal of Forest Research 15, 1055-1060.

Lu, Z., Zhang, Q., Streets, D.G. (2011) Sulfur dioxide and primary carbonaceous aerosol emission in China and India, 1996-2010. Atmospheric Chemistry and Physics 11, 9839-9864.

Matsuda, K., Fujimura, Y., Hayashi, K., Takahashi, A., Nakaya, K. (2010) Deposition velocity of PM2.5 sulfate in the summer above a deciduous forest in central Japan. Atmospheric Environment 44, 4582-4587.

Matsuda, K., Watanabe, I., Wingpud, V., Theramongkol, P., Ohizumi, T. (2006) Deposition velocity of $\mathrm{O}_{3}$ and $\mathrm{SO}_{2}$ in the dry and wet season above a tropical forest in northern Thailand. Atmospheric Environment 40, 7557-7564.

Matsuda, K., Sase, H., Murao, N., Fukazawa, T., Khoomsub, K., Chanonmuang, P., Visaratana, T., Khummongkol, P. (2012) Dry and wet deposition of elemental carbon on a tropical forest in Thailand. Atmospheric Environment 54, 282-287.

Mayer, R. (1993) Filtering of air-borne contaminants by vegetation canopies. In Soil Monitoring (Schulin, R., Desaules, A., Webster, R., von Steiger, B. Eds), Birkhäuser Verlag, Basel, pp. 89-103.

Network Center for EANET (2010) Report of the Interlaboratory Comparison Project 2010. Asia Center for Air Pollution Research, Niigata, Japan.

Ohara, T., Akimoto, H., Kurokawa, J., Horii, N., Yamaji, K., Yan, X., Hayasaka, T. (2007) An Asian emission inventory of anthropogenic emission sources for the period 1980-2020. Atmospheric Chemistry and Physics 7, 4419-4444.

Ohizumi, T., Take, N., Moriyama, N., Suzuki, O., Kusakabe, M. (2001). Seasonal and spatial variations in the chemical and sulfur isotopic composition of acid depo- sition in Niigata Prefecture, Japan. Water, Air, and Soil Pollution 130, 1679-1684.

Rautio, P., Huttunen, S. (2003) Total vs. internal element concentrations in Scots pine needles along a sulphur and metal pollution gradient. Environmental Pollution 122, 273-289.

Robertson, A.L., Malhi, Y., Farfen-Amezquita, F., Eduardo, L., Aragão, C., Espejo, J.E.S., Robertson, M.A. (2010) Stem respiration in tropical forests along an elevation gradient in the Amazon and Andes. Global Change Biology 16, 3193-3204.

Sanhueza, E., Elbert, W., Rondon, A., Arias, M.C., Hermeso, M. (1989) Organic and inorganic acids in rain from a remote site of the Venezuelan savannah. Tellus 41B, 170-176.

Sanhueza, E., Arias, M.C., Donoso, L., Graterol, N., Hermoso, M. Marti, I., Romero, J., Rondon, A., Santana, M. (1992) Chemical composition of acid rains in the Venezuelan savannah region. Tellus 44B, 54-62.

Sase, H., Takamatsu, T. (2009) Atmospheric deposition and its leaf surface interactions in Japanese cedar forests. In Forest Canopies: Forest Production, Ecosystem Health and Climate Conditions (Creighton, J.D. and Roney, P.J. Eds). Nova Science Publishers. pp. 127-141.

Sase, H., Takamatsu, T., Yoshida, T., Inubushi, K. (1998) Changes in properties of epicuticular wax and the related water loss in Japanese cedar (Cryptomeria japonica) affected by anthropogenic environmental factors. Canadian Journal of Forest Research 28, 546-556.

Sase, H., Takahashi, A., Sato, M., Kobayashi, H., Nakata, M., Totsuka, T. (2008) Seasonal variation in the atmospheric deposition of inorganic constituents and canopy interactions in a Japanese cedar forest. Environmental Pollution 152, 1-10.

Shibata, H., Sakuma, T. (1996) Canopy modification of precipitation chemistry in deciduous and coniferous forests affected by acidic deposition. Soil Science and Plant Nutrition 42, 1-10.

Staelens, J., Houle, D., De Schrijver, A., Neirynck, J., Verheyen, K. (2008) Calculating dry deposition and canopy exchange with the canopy budget model: Review of assumption and application to two deciduous forests. Water, Air and Soil Pollution 191, 149-169.

Takamatsu, T., Sase, H., Takada, J. (2001a) Some physiological properties of Cryptomeria japonica leaves from Kanto, Japan: potential factors causing tree decline. Canadian Journal of Forest Research 31, 663-672.

Takamatsu, T., Sase, H., Takada, J., Matsushita, R. (2001b) Annual changes in some physiological properties of Cryptomeria japonica leaves from Kanto. Water, Air and Soil Pollution 130, 941-946.

Takamatsu, T., Takada, J., Matsushita, R., Sase, H. (2000) Aerosol elements on tree leaves -Antimony as a possible indicator of air pollution-. Global Environmental Research 4(1), 49-60.

Turunen, M., Huttunen, S. (1990) A review of the response of epicuticular wax of conifer needles to air pollution. Journal of Environmental Quallity 19, 35-45. 
Wachirinrat, C., Takeda, H. (2003) Litterfall patterns of dry deciduous and evergreen forests in Sakaerat and vicinity area, northeastern Thailand. In Final report for NRCT, Decomposition and nutrient cycling processes in tropical seasonal forests in Thailand (Takeda, H., Khamyong, S., Wiwatwitaya, D. Eds), Kyoto University, Kyoto, pp. 7-23.

Yamashita, N., Ohta, S., Sase, H., Luangjame, J., Visara- tana, T., Kietvuttinon, B., Garivait, H., Kanzaki, M. (2010) Seasonal and spatial variation of nitrogen dynamics in the litter and surface soil layers on a tropical dry evergreen forest slope. Forest Ecology and Management 259, 1502-1512.

(Received 30 May 2012, revised 26 July 2012, accepted 19 September 2012) 